\title{
Incontinência urinária no puerpério de parto vaginal e cesárea: revisão de literatura
}

\author{
Urinary incontinence in postpartum of vaginal delivery and cesarean: literature review
}

\author{
Alice Rios Borba ${ }^{1}$, Daniela Kich Brondani², Mara Regina Knorst ${ }^{3}$
}

\section{RESUMO}

Objetivo: O estudo objetivou revisar a literatura quanto à presença de incontinência urinária (IU) no puerpério de parto normal e cesárea.

Materiais e Métodos: Foi realizada uma pesquisa nos bancos de dados PubMed/MedLine, COCHRANE e EMBASE. Foram selecionados os seguintes tipos de estudo: prospectivo multicêntrico, caso-controle, prospectivo, coorte, multicêntrico prospectivo observacional, randomizado controlado, prospectivo observacional, coorte controlado, transversal, base populacional, publicados no período de 2002 a 2012, nos idiomas inglês e português, em que as palavras-chave estivessem no título ou resumo. As palavras-chave utilizadas foram: "parto vaginal", "cesárea" e "incontinência urinária".

Resultados: Os 14 estudos analisados, avaliaram a presença de IU no puerpério de parto vaginal e cesárea. Observamos que a cesárea para ter seu papel protetor no aparecimento da IU no puerpério deve ser realizada de maneira eletiva. Os distúrbios do assoalho pélvico estão fortemente ligados com idade materna, fumo, obesidade, parto de fórceps, multiparidade, episiotomia, duração da segunda fase do trabalho de parto, gravidez e constipação crônica. Conclusão: Conclui-se que a segunda fase prolongada do trabalho de parto tem papel significativo na presença da IU no puerpério em ambos modos de parto, e que a cesárea teria o seu valor protetor no desenvolvimento da IU no puerpério apenas se fosse realizada de maneira eletiva. Dos 14 artigos, apenas um estudo foi realizado na população brasileira, mostrando a importância de serem realizados mais estudos sobre IU no puerpério no Brasil.

Palavras-chave: incontinência urinária; parto normal; cesárea.

\section{ABSTRACT}

Objective: The present study aimed to review the literature regarding the presence of urinary incontinence (UI) in postpartum of vaginal delivery and cesarean section.

Materials and Methods: A survey was conducted in the databases PubMed/Medline, COCHRANE and EMBASE, which were selected the following types of study: prospective multicenter study, case-control, prospective study, cohort study, prospective observational multicenter study, randomized controlled study, prospective observational study, cohort study controlled, cross-sectional, population-based, published in the period 2002-2012, in english and portuguese, where the keywords were in the title or abstract. The keywords used are: "vaginal delivery", "cesarean" and "urinary incontinence". Results: The 14 studies analyzed evaluated the presence of UI in postpartum of vaginal delivery and cesarean. We observed that cesarean section to have your protective role in the appearance of the UI in the puerperium must be performed electively. The pelvic floor disorders are linked with maternal age, smoking, obesity, forceps, multiparity, episiotomy, second stage of labor, pregnancy and chronic constipation.

Conclusions: It is concluded that prolonged second stage of labor has a significant role in the presence of UI in the puerperium in both modes of delivery, and cesarean section would have its protective value in the development of UI puerperal only if performed electively. Of the 14 selected articles, only one study was conducted in the brazilian population, showing the importance of further studies on UI postpartum in Brazil.

Keywords: urinary incontinence; vaginal delivery; cesarean delivery.

\footnotetext{
${ }^{1}$ Fisioterapeuta formada pela Pontifícia Universidade Católica do Rio Grande do Sul (PUCRS).

${ }^{2}$ Acadêmica do Curso de Fisioterapia da PUCRS.

${ }^{3}$ Fisioterapeuta. Doutora em Gerontologia Biomédica pela PUCRS. Professora do Curso de Fisioterapia da PUCRS.
} 


\section{INTRODUÇÃO}

Incontinência urinária (IU) é definida como qualquer perda involuntária de urina $^{1}$, é mais comum em mulheres do que em homens e sua prevalência aumenta com a idade ${ }^{2}$. A prevalência varia de $3,6 \%$ a $15 \%$ antes da gravidez, e de $28 \%$ a $64 \%$ durante a gravidez ${ }^{3}$.

A IU é classificada em IU de esforço (IUE), que ocorre durante tosse, espirro ou esforços físicos; IU de urgência (IUU), que ocorre conjuntamente a um desejo imperioso de urinar; e IU mista (IUM), associada com ambos sintomas 4 .

Na população de gestantes jovens, o risco, incidência e prevalência de IUE aumenta, e os maiores fatores de risco para o aparecimento da IU seriam: idade, influências hormonais, obesidade, diabetes mellitus, gravidez e parto, cirurgia pélvica, medicações e fatores genéticos ${ }^{5}$. Entretanto, há relato que tanto a gravidez como o parto geram mudanças na anatomia e na função do assoalho pélvico e que certas disfunções poderiam ser causadas tanto por traumas visíveis no momento do parto como por danos escondidos em nervos e músculos ${ }^{6}$.

A gravidez é um episódio fisiológico na vida da mulher, levando a transformações em seu corpo que não seriam normais fora do ciclo gestatório. Tais transformações são necessárias para que o embrião possa ser concebido e se desenvolver. Todo o ciclo gestatório dura em média 40 semanas, o equivalente a 280 dias $^{1}$.

Durante a gestação inúmeras alterações ocorrem no sistema urinário. Ambos os ureteres e pelves renais têm grande dilatação, que inicia precocemente com 10 semanas. Já a bexiga é gradativamente elevada pelo útero que está em crescimento, principalmente nos dois últimos trimestres ${ }^{7}$.

O retorno da função renal em nível pré-gestacional ocorre em 6 meses no pós-parto. Já as alterações morfológicas do trato urinário, como a dilatação da uretra, pelve renal e ureteres, e o relaxamento da parede vesical podem persistir por até 3 meses após o parto ${ }^{8}$.

O parto normal pode desencadear IU devido ao risco de lesões aos músculos e nervos, às estruturas conectivas que suportam a pelve, às estruturas vasculares, ou diretamente ao trato urinário ${ }^{9}$. Além do parto, a episiotomia é motivo de várias controvérsias com relação à sua real eficácia na proteção das lesões perineais, devido ao aumento da dor e das complicações intra e pós-operatórias ${ }^{10}$.

Além da via de parto vaginal, alterações anatômicas e fisiológicas que ocorrem no trato urinário inferior ao longo da gestação predispõem o desencadeamento de Sintomas Urinários Irritativos (SUI). A cesareana eletiva tem sido associada ao menor risco de incidência de sintomas urinários, quando comparada ao parto vaginal. Entretanto, o efeito protetor da cesareana eletiva, a longo prazo, permanece controverso ${ }^{11}$.

O presente estudo objetivou revisar a literatura quanto à presença de IU no puerpério de parto normal e cesárea.

\section{MATERIAIS E MÉTODOS}

Este estudo consiste em uma revisão bibliográfica realizada através de pesquisa nos bancos de dados PubMed/MedLine, COCHRANE e EMBASE. Foram selecionados os seguintes tipos de estudo: estudo prospectivo multicêntrico, caso-controle, estudo prospectivo, estudo de coorte, estudo multicêntrico prospectivo observacional, estudo randomizado controlado, estudo prospectivo observacional, estudo coorte controlado, estudo transversal, estudo de base populacional, publicados no período de 2002 a 2012, no idioma inglês ou português, em que as palavras-chave estivessem no título ou no resumo. As palavras-chave utilizadas foram: "parto vaginal", "cesárea" e "incontinência urinária", sendo pesquisadas também com a terminologia em inglês "vaginal delivery", "cesarean" e "urinary incontinence". Foram excluídos dessa revisão artigos que continham homens na pesquisa, os que não apresentavam o número de participantes, aqueles em que as pacientes foram submetidas a algum tipo de intervenção cirúrgica ou fisioterapêutica durante o estudo e os que não apresentavam delineamento.

Após a seleção dos artigos, foi realizada a leitura dos mesmos e os dados analisados pelas pesquisadoras, através das leituras exploratória, seletiva, analítica e 
TABELA 1 - Número de participantes, delineamento e instrumentos.

\begin{tabular}{|c|c|c|c|}
\hline Autor/Ano & $\mathbf{N}$ & Delineamento & Instrumento \\
\hline Torrisi et al. ${ }^{17}$ (2012) & 960 & Estudo prospectivo multicêntrico & $\begin{array}{l}\text { Questionário ICIQ-SF escala Wexner's de } \\
\text { classificação de continência }\end{array}$ \\
\hline Torkestani et al. ${ }^{22}$ (2009) & 250 & Caso-controle & $\begin{array}{l}\text { Questionário e exame físico, exame através do } \\
\text { POPQ (Pelvic Organ Prolapse Quantification) }\end{array}$ \\
\hline Groutz et al. ${ }^{19}(2004)$ & 363 & Estudo prospectivo & $\begin{array}{c}\text { Questionário e coleta de dados do prontuário } \\
\text { hospitalar }\end{array}$ \\
\hline Brown et al. ${ }^{26}(2011)$ & 1507 & Estudo coorte & Entrevista por telefone e questionário \\
\hline $\begin{array}{l}\text { Van Brummen et al. }{ }^{18} \\
(2007)\end{array}$ & 344 & Estudo coorte & Questionário \\
\hline McKinnie et al. ${ }^{23}$ (2005) & 978 & $\begin{array}{l}\text { Estudo multicêntrico prospectivo } \\
\text { observacional }\end{array}$ & Questionário \\
\hline Foldspang et al. ${ }^{16}$ (2004) & 1232 & Estudo randomizado controlado & Questionário \\
\hline Arrue et al. ${ }^{13}(2010)$ & 330 & Estudo prospectivo & Questionário ICIQ-SF, entrevista e perineometria \\
\hline Lorzadeh et al. ${ }^{20}$ (2009) & 330 & Estudo prospectivo & Questionário \\
\hline Shek et al. ${ }^{24}$ (2011) & 367 & Estudo prospectivo observacional & Entrevista, ultrassom e tomografia \\
\hline Ferederice et al. ${ }^{25}$ (2011) & 46 & Estudo coorte & Exame físico e coleta de dados do prontuário \\
\hline Ekström et al. ${ }^{15}(2008)$ & 435 & Estudo coorte controlado & Questionário \\
\hline Lukacz et al. ${ }^{21}$ (2006) & 3050 & Estudo transversal & Questionário EPIQ \\
\hline Boyles et al. ${ }^{14}$ (2009) & 5599 & Estudo de base populacional & Questionário \\
\hline
\end{tabular}

interpretativa $^{12}$.

\section{RESULTADOS E DISCUSSÃO}

A busca inicial identificou 651 artigos, dos quais 34 foram recuperados para leitura na íntegra, restando ao final 14 artigos. A figura 1 demonstra o fluxograma dos estudos incluídos.

Os estudos incluídos nesta revisão analisaram a presença da IU no puerpério de parto vaginal e cesárea. A tabela 2 resume os principais resultados dos estudos incluídos.

A maioria dos estudos sugere uma relação direta entre parto vaginal e a presença de IU no período puerperal ${ }^{13-18}$. Um estudo relatou que após o nascimento, a IUE foi significantemente mais prevalente no grupo do parto vaginal ${ }^{17}$. Há de se considerar também que todos os estudos que sugerem que o parto normal está relacionado com IU no puerpério, relacionam a presença da mesma durante a gestação ${ }^{13-}$ 18.

Foi constatado em uma pesquisa que a IU na gestação se manifestou por si só como uma crucial e independente precursora de IU no puerpério ${ }^{16}$. O uso do fórceps e o peso maior do recém-nascido ( $>=4000 \mathrm{~g}$ ) parecem acentuar a tensão no assoalho pélvico durante o período expulsivo, promovendo lesões nas suas estruturas, inclusive no esfíncter anal ${ }^{11}$.

Além disso, temos a presença em dois estudos de coorte, com 435 e o outro com 344 participantes respectivamente, da comparação da incidência de IU entre parto vaginal e cesárea, associando o parto vaginal com o aumento significativo da prevalência de IUE quando comparado a cesárea eletiva em mulheres primíparas $^{15,18}$.

Também foram encontrados indícios de que o risco de desordens do assoalho pélvico é menor com a cesárea 
TABELA 2 - Principais resultados encontrados.

\begin{tabular}{|c|c|}
\hline Autor/Ano & Principais resultados \\
\hline Torrisi et al. ${ }^{17}$ (2012) & $\begin{array}{l}\text { Início de IU e fecal durante a gestação, história familiar positiva e parto vaginal são fatores de } \\
\text { risco independentes para a persistência dos sintomas de IU e fecal no pós-parto imediato. }\end{array}$ \\
\hline Torkestani et al. ${ }^{22}$ (2009) & $\begin{array}{l}\text { Relação significativa entre IU e gestação, paridade, tipo de parto, episiotomia, tipo de cesárea, } \\
\text { cistocele, retocele e constipação crônica. Não há relação entre IU e o peso do bebê ao nascer, } \\
\text { ou o tipo de trabalho da mãe. Cesárea pode diminuir a taxa de IU. }\end{array}$ \\
\hline Groutz et al. ${ }^{19}(2004)$ & $\begin{array}{l}\text { Prevalência da IUE no pós-parto é semelhante após o parto vaginal e a cesárea realizada em } \\
\text { trabalho de parto obstruído. Cesárea eletiva foi associada a uma prevalência significativamente } \\
\text { menor de IUE. }\end{array}$ \\
\hline Brown et al. ${ }^{26}$ (2011) & $\begin{array}{l}\text { Além da própria gravidez, as alterações fisiológicas associadas com a segunda fase do trabalho } \\
\text { de parto parecem desempenhar um papel na IU pós-parto. }\end{array}$ \\
\hline Van Brummen et al. ${ }^{18}$ (2007) & $\begin{array}{l}\text { IUE foi mais prevalente no grupo do parto vaginal. No parto vaginal e cesárea a presença de } \\
\text { IUE no início da gravidez aumenta o risco da mesma um ano após o parto. }\end{array}$ \\
\hline McKinnie et al. ${ }^{23}$ (2005) & $\begin{array}{l}\text { A gravidez aumenta o risco de IU e fecal. Cesárea não diminui o risco de IU e fecal quando } \\
\text { comparada ao parto vaginal. }\end{array}$ \\
\hline Foldspang et al. ${ }^{16}$ (2004) & $\begin{array}{l}\text { Maiores riscos de IU no puerpério foram achados entre mulheres que queixavam-se de IU } \\
\text { durante a gravidez. Alto número de partos vaginais sugere IU atribuível ao parto vaginal. }\end{array}$ \\
\hline Arrue et al. ${ }^{13}(2010)$ & $\begin{array}{l}\text { IUE leve ou moderada foi comum após o primeiro parto vaginal. IU durante a gestação foi o } \\
\text { único fator independentemente associado com a presença de IUE } 6 \text { meses após o parto vaginal. }\end{array}$ \\
\hline Lorzadeh et al. ${ }^{20}$ (2009) & $\begin{array}{l}\text { Frequência de IUE é similar no parto vaginal espontâneo e na cesárea após um trabalho de } \\
\text { parto obstruído. Cesárea eletiva está associada com um risco menor de IUE. }\end{array}$ \\
\hline Shek et al. ${ }^{24}$ (2011) & $\begin{array}{l}\text { Parece haver um aumento generalizado na mobilidade uretral após o parto, mas essa mudança } \\
\text { não parece estar associada com IUE. }\end{array}$ \\
\hline Ferederice et al. ${ }^{25}$ (2011) & $\begin{array}{l}\text { Prevalência de sintomas urinários } 60 \text { dias pós-parto vaginal com episiotomia e cesareana após } \\
\text { trabalho de parto é baixa. Não há associação entre a função muscular do assoalho pélvico e } \\
\text { sintomas urinários. }\end{array}$ \\
\hline Ekström et al. ${ }^{15}$ (2008) & $\begin{array}{l}\text { Parto vaginal está associado com o aumento da prevalência de IUE quando comparado a } \\
\text { cesárea eletiva em primíparas. Sinais no final da gravidez ou } 3 \text { meses pós-parto são risco dos } \\
\text { sintomas se agravarem nos } 9 \text { meses pós-parto vaginal e cesárea obstruída. }\end{array}$ \\
\hline Lukacz et al. ${ }^{21}$ (2006) & $\begin{array}{l}\text { Risco de desordens do assoalho pélvico está independentemente associado com o parto } \\
\text { vaginal, mas não com a paridade. A cesárea tem um efeito protetor similar a nuliparidade no } \\
\text { desenvolvimento das desordens do assoalho pélvico quando comparadas ao parto vaginal. }\end{array}$ \\
\hline Boyles et al. ${ }^{14}$ (2009) & $\begin{array}{l}\text { IU é comum no pós-parto imediato após a primeira gravidez. Parto vaginal aumenta o risco de } \\
\text { IU. }\end{array}$ \\
\hline
\end{tabular}

quando comparada com o parto vaginal, levando a crer que a cesárea teria um efeito protetor, similar a nuliparidade, no desenvolvimento dessas desordens ${ }^{19-}$ 22. Em 2009 um estudo com 250 mulheres, visou investigar o papel protetor de cesárea na IU e os resultados obtidos sugeriram que a cesárea pode diminuir a taxa de incidência da incontinência ${ }^{22}$. Groutz et al. ${ }^{19}$, concordando com o estudo anterior, faz a ressalva de que a cesárea, para ter seu papel protetor, deve ser realizada de maneira eletiva. Em contrapartida, ainda no mesmo estudo, o autor também leva em consideração que a cesárea pode expor as mulheres a uma maior morbi-mortalidade.

Discordando dos estudos citados até o momento, apenas dois autores não se posicionaram contra o parto vaginal, levando a entender que esse modo de parto não está relacionado diretamente com a IU no puerpério ${ }^{23,24}$. Já é comprovado que há um aumento generalizado na mobilidade uretral após o parto, mas pesquisas mostram que esta mudança não parece estar associada com IUE ${ }^{24}$. Um estudo acompanhou 1004 mulheres durante 18 meses, mostrando que pacientes que realizaram exclusivamente cesárea eletiva não apresentaram diminuição significante de IU ou fecal comparadas com aquelas que realizaram pelo menos um parto vaginal a termo ${ }^{23}$.

No entanto, um estudo em particular, realizado com 46 participantes, demonstrou que a prevalência dos SUI 


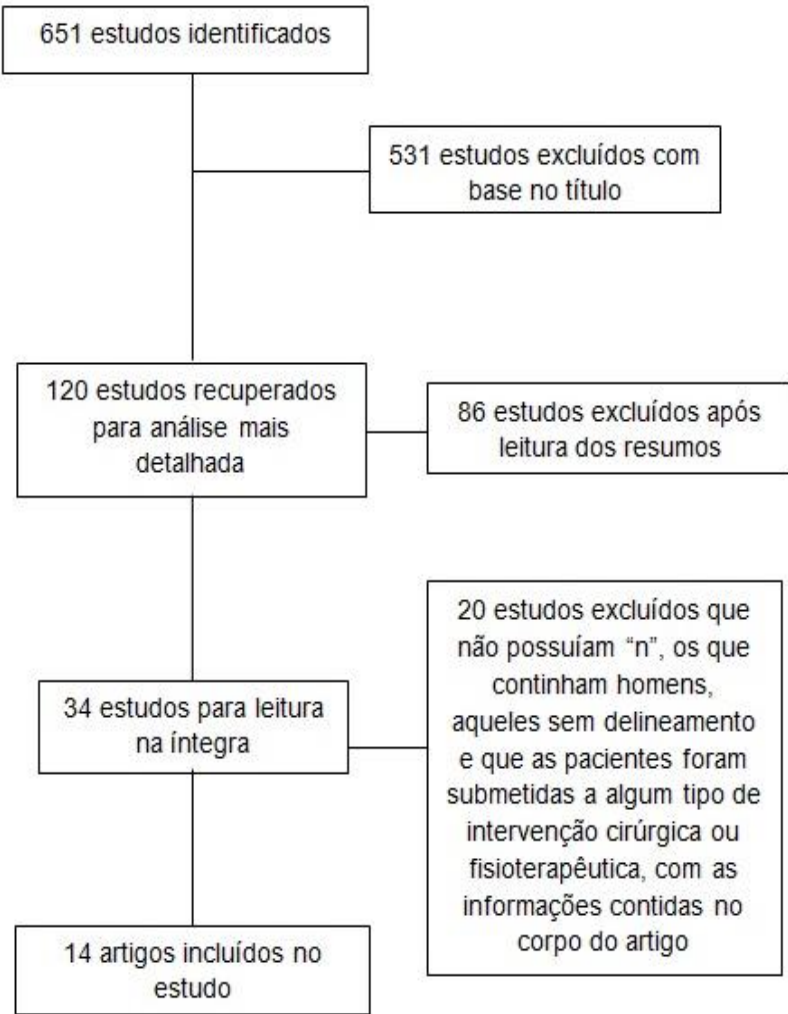

FIGURA 1 - Fluxograma dos estudos incluídos na revisão.

60 dias pós-parto vaginal e cesárea realizada após o início do trabalho de parto é baixa, e não foi demonstrada associação entre a função muscular do assoalho pélvico e sintomas urinários. Este foi o único estudo encontrado que não se posicionou a favor ou contra nenhum dos modos de parto ${ }^{25}$.

A conclusão do acompanhamento de 1507 mulheres em um estudo coorte mostrou que os maiores riscos de IU pós-parto foram associados com uma segunda fase prolongada do trabalho de parto, independentemente do nascimento ter sido espontâneo ou com o auxílio de fórceps ou vácuo. O autor menciona ainda que a cesárea pareceu reduzir o risco de IU, mas $10 \%$ das mulheres que realizaram cesárea eletiva/ou na primeira fase do trabalho de parto, e $20 \%$ das que realizaram cesárea de emergência, relataram sintomas nos primeiros três meses pós-parto ${ }^{26}$.

Os distúrbios do assoalho pélvico estão fortemente ligados com a idade materna, fumo, obesidade, parto de fórceps, multiparidade, episiotomia, duração da segunda fase do trabalho de parto, gravidez e constipação crônica ${ }^{22}$. Torrisi et al. ${ }^{17}$ relata em seu estudo que a história familiar de IU e incontinência anal está associada ao aparecimento da IU três meses após o parto. Enquanto Ferederice et al. ${ }^{25}$ menciona que puérperas obesas e com sobrepeso tiveram 4,6 vezes mais queixas de noctúria, urgência e aumento da frequência urinária diurna, nos levando a observar que o peso da puérpera pode também ser um fator predisponente da IU.

A maioria dos artigos incluídos em nosso estudo, apresentou que a segunda fase prolongada do trabalho de parto é um fator predisponente para o desencadeamento da IU, e que a cesárea seria um fator protetor, se fosse realizada de maneira eletiva ${ }^{13,17,18,19,25,26}$. Mulheres que realizaram parto vaginal, após uma segunda fase prolongada do trabalho de parto, têm grande probabilidade de apresentar IU nos primeiros 3 meses pós-parto, quando comparadas com mulheres que não tiveram a segunda fase prolongada, o mesmo acontece com mulheres que fizeram cesareana após a segunda fase prolongada do trabalho de parto $^{26}$.

Através dos estudos analisados conclui-se que a segunda fase prolongada do trabalho de parto tem papel significativo na presença da IU no puerpério em ambos modos de parto, e que a cesárea teria o seu valor protetor no desenvolvimento da IU no puerpério, apenas se fosse realizada de maneira eletiva. Dos 14 artigos selecionados apenas um estudo foi realizado na população brasileira ${ }^{25}$, mostrando a importância de serem realizados mais estudos sobre IU no puerpério no Brasil.

\section{REFERÊNCIAS}

1. Knorst MR, Cavazotto $\mathrm{K}$, Henrique M, Resende TL. Intervenção fisioterapêutica em mulheres com incontinência urinária associada ao prolapso de órgão pélvico. Rev Bras Fisioter. 2012;16(2):102-7.

2. Adams E, Bardsley A, Crumlin L, Currie I, Evans L, Haslam J. Urinary incontinence the management of urinary incontinence in women. Published by the RCOG Press at the Royal College of Obstetricians and Gynaecologists, 27 Sussex Place, Regent's Park, London NW1 4RG. October 2006. [acesso em 2012 outubro 28]. Disponível em: http://www.nice.org.uk/nicemedia/pdf/CG40fullguideline.pd f

3. Santos PC, Mendonça D, Alves O, Barbosa AM. Prevalência e impacte da incontinência urinária de stresse: antes e durante a gravidez. Acta Med Port. 2006;19:349-56.

4. Haylen BT, de Ridder D, Freeman RM, Swift SE, Berghmans B, Lee J, Monga A, Petri E, Rizk DE, Sand PK, Schaer GN. An International Urogynecological 
Association (IUGA)/International Continence Society (ICS) joint report on the terminology for female pelvic floor dysfunctions. Neurourol Urodyn. 2010;29(1):4-20.

5. Stothers L, Friedman B. Risk factors for the Development of Stress Urinary Incontinence in Women. Curr Urol Rep. 2011;12:363-69.

6. Rogers RG, Leeman LM, Migliaccio L, Albers LL. Does the severity of spontaneous genital tract trauma affect postpartum pelvic floor function. Int Urogynecol J. 2008;19:429-35.

7. Scarpa KP, Herrmann V, Palma PCR, Ricetto CLZ, Morais $S$. Prevalência de sintomas urinários no terceiro trimestre da gestação. Rev Assoc Med Bras. 2006;52(3):153-6.

8. Baracho E. Fisioterapia aplicada à obstetrícia: aspectos de Ginecologia e Neonatologia. $3^{\underline{a}}$ ed. Rio de Janeiro: MEDS Editora Médica; 2002

9. Baracho SM. Preditores de Incontinência Urinária de Esforço em mulheres primíparas que realizaram parto vaginal [dissertação]. Belo Horizonte (MG): Universidade Federal de Minas Gerais; 2010.

10. Carvalho CCM, Souza ASR, Moraes Filho OB. Episiotomia seletiva: avanços baseados em evidências. FEMINA 2010;38(5):265-70.

11. Scarpa KP, Herrman V, Palma PCR, Riccetto CLZ, Morais S. Sintomas urinários irritativos após parto vaginal ou cesárea. Rev Assoc Med Bras. 2009;55(4):416-20.

12. Gil AC. Como delinear uma pesquisa bibliográfica In: Gil AC. Como elaborar projetos de pesquisa. São Paulo: Atlas; 2002. p. 59-85.

13. Arrue $M$, Ibañez $L$, Paredes $J$, Murgiondo $A$, Blear $M$, Sarasqueta C, Diez-Itza I. Stress urinary incontinence six months after first vaginal delivery. EJOG. 2010;150:210-4.

14. Boyles SM, Li H, Mori T, Osterweil P, Guise JM. Effect of mode of delivery on the incidence of urinary incontinence in primiparous women. Obstet Gynecol. 2009;113:134-41.

15. Ekström A, Altman D, Wiklund I, Larsson C, Andolf E. Planned cesarean section versus planned vaginal delivery: comparison of lower urinary tract symptoms. Int Urogynecol J. 2008;19:459-65.

16. Foldspang A, Wvidman L, Mommsen S, Nielsen JB. Risk of postpartum urinary incontinence associated with pregnancy and mode of delivery. Acta Obtet Gynecol Scand. 2004;83:923-7.

17. Torrisi G, Minini G, Bernasconi F, Perrone A, Trezza G Guardabasso V, Ettore G. A prospective study of pelvic floor dysfunctions related to delivery. EJOG. 2012;160:110-5.

18. Van Brummen $H J$, Bruinse HW, Van de Pol G, Heintz APM, Van der Vaart $\mathrm{CH}$. The effect of vaginal and cesarean deliver on lower urinary tract symptoms: what makes the difference?. Int Urogynecol J. 2007;18:133-9.

19. Groutz A, Rimon E, Peled S, Gold R, Pauzner D, Lessing JB, Gordon D. Cesarean section: Does it really prevent the development of postpartum stress urinary incontinence? A prospective study of 363 women one year after their first delivery. Neurol Urodynam. 2004;23:2-6.

20. Lorzadeh N, Kazemirad S. The effect of cesarean section in preventing postpartum stress urinary incontinence in primiparous women after one year of delivery. Res $J$ Obstet Gynecol. 2009;2(1):1-5.

21. Lukacz ES, Lawrence JM, Contreras R, Nager CW, Luber KM. Parity, mode of delivery, and pelvic floor disorders. Obstet Gynecol. 2006;107:1253-60.

22. Torkestani F, Zafarghandi N, Davati A, Hadavand SH, Garshasbi M. Case-controlled study of the relationship between delivery method and incidence of post-partum urinary incontinence. JIMR. 2009;37:214-9.

23. McKinnie V, Swift SE, Wang W, Woodman P, O'boyle A, Kahn M, Valley M, Bland D, Schaffer J. The effect of pregnancy and mode of delivery on the prevalence of urinary and fecal incontinence. AJOG. 2005;193:512-8.

24. Shek KL, Dietz HP, Kirby A. The effect of childbirth on urethral mobility: a prospective observational study. J Urol. 2010;184:629-34.

25. Ferederice $\mathrm{CP}$, Amaral E, Ferreira NO. Urinary symptoms and the pelvic floor muscle function after delivery. Rev Bras Gynecol Obstet. 2011;33(4):188-95.

26. Brown SJ, Gartland D, Donath S, MacArthur C. Effects of prolonged second stage, method of birth, timing of cesarean section and other obstetrics risk factors on postnatal UI. BJOG. 2011;118:991-1000.

\section{Endereço para correspondência}

Alice Rios Borba

Rua Antônio Pinheiro Cabral $n^{\circ} 151$

Porto Alegre/RS - CEP 91755-112

Telefone: +55 5198220245

E-mail: rios alice@live.com 